\title{
Formulation and Evaluation of Gastro Retentive Floating Tablets of Simvastatin using Hydrophilic Rate Retardant
}

\author{
Md. Nazmul Hussain ${ }^{1}$, Md. Abdullah Al Masum², Sharmin Akhter², Florida Sharmin² and Md. Selim Reza' \\ ${ }^{1}$ Department of Pharmaceutical Technology, Faculty of Pharmacy, University of Dhaka, Dhaka-1000, Bangladesh \\ ${ }^{2}$ Department of Pharmacy, University of Asia Pacific, Dhanmondi, Dhaka-1209, Bangladesh
}

\begin{abstract}
Gastro retentive floating tablet of Simvastatin was prepared by direct compression technique using Methocel K4M as the rate controlling polymer. The hydrophilic cellulose derivative, Methocel K4M was evaluated for its gel forming and release controlling properties. Sodium bicarbonate and citric acid were incorporated as gas generating agents. The effects of soluble components (sodium bicarbonate and citric acid) and gel forming agents on drug release profile and floating properties were investigated. The tablets from all formulations were evaluated for thickness, diameter, weight variation, hardness, and friability. The tablets were also tested for the buoyancy studies and in vitro drug release studies. The drug release study was evaluated for eight hours using USP XXII paddle-type dissolution apparatus using $0.1 \mathrm{~N} \mathrm{HCl}$ with $1 \%$ Sodium Lauryl Sulphate as dissolution medium. The release mechanisms were explored and explained with zero order, first order, Higuchi, Hixon Crowell and Korsmeyer equations. The release rate, extent and mechanisms were found to be governed by the polymer content. It was found that the mean dissolution time, percentage drug release, release rate constant and diffusion exponent were influenced significantly by the amount of polymer incorporation.
\end{abstract}

Keywords: Floating tablets, buoyancy, sustained release, hydrophilic polymer, release exponent and Simvastatin.

\section{Introduction}

High level of patient compliance has been observed in taking oral dosage forms is due to the ease of administration and handling of these forms. A lot of advancements have been seen in oral controlled drug delivery system in the last few decades. But still oral sustained drug delivery system is complicated by limited gastric residence time. Rapid GI transit can prevent complete drug release in the absorption zone and reduce the efficacy of the administered dose since the majority of drugs are absorbed in stomach or the upper part of small intestine (Choi et al., 2002; Rouge et al., 1996). To overcome these limitations, various approaches have been proposed to increase gastric residence of drug delivery systems in the upper part of the gastrointestinal tract like floating drug dosage systems (FDDS) (Singh and Kim, 2000). Gastroretentive systems can remain in the gastric region for several hours and hence can significantly prolong the gastric residence time of drugs that offer numerous advantages; improves bioavailability, reduces drug waste and improves solubility for drugs that are less soluble in a high $\mathrm{pH}$ environment of small intestine (Gendle et al., 2010).

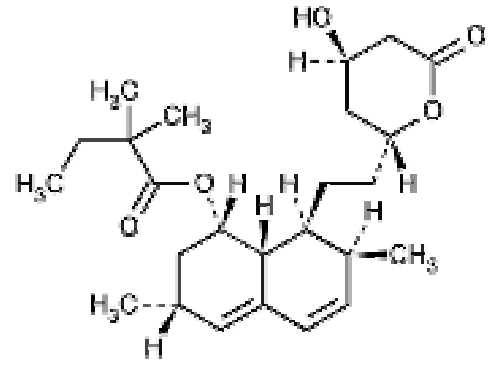

Simvastatin, a 3-hydroxy-3-methylglutaryl coenzyme A (HMG-CoA) reductase inhibitor (the structure is given above), is a statin with well-known lipid lowering effects that substantially decrease cardiovascular morbidity and mortality in patients with and without coronary artery diseases (Ganesh et al., ,2003; LaRosa, 2000). Recently it has been reported to simulate bone formation both in vivo and in vitro animal osteoporosis models (Mundy et al., 1999). But Simvastatin is accused for extensive excretion after oral administration; $60 \%$ of the dose is excreted by feces after ingestion. Besides, Simvastatin undergoes extensive first-pass extraction in the liver and hence, the availability of the drug to the general circulation is very low (below 5\%). Therefore, gastro retentive drug delivery system might be advantageous for Simvastatin. 
Statins are good candidate for gastro retentive drug delivery system. Therefore, various researches have been reported on gastro retentive drug delivery system of Statins to overcome the problems associated with oral administration. Floating tablet of Fluvastatin was prepared by Asif et al., (2010), Lovastatin and Atenolol were combined in floating tablet by Kulkarni and Bhatia, (2009) and Sharmin et al., (2011) reported gastro retentive drug delivery system of Atorvastatin Calcium. With a goal to improve the absorption and oral bioavailability, we took an attempt to formulate floating drug delivery systems using Simvastatin as the model drug with a hydrophilic cellulose derivative e Methocel K4M. Investigations were performed to observe the effect of polymer content upon the floating lag time of the tablets. The impact of polymer loading upon the release rate, mean dissolution time and release mechanism were also evaluated with the help of various mathematical models.

\section{Materials and Methods}

Materials: Simvastatin and Methocel K4M were obtained from Incepta Pharmaceuticals Ltd, Bangladesh and Colorcon, USA respectively as generous gift sample. Polyvinylpyrrolidone K-30 and two gas generating agents citric acid and sodium bi-carbonate were obtained from ACI Pharmaceuticals Ltd, Bangladesh. All other ingredients were of analytical grade and purchased from local market.

Preparation of floating tablets of simvastatin: The active ingredient and other excipients were accurately weighed for 50 tablets according to the formulations (Table 1). Particular attention has been given to ensure thorough mixing and phase homogenization. The appropriate amounts of the mixture were accurately weighed in an electronic balance for the preparation of each tablet and finally compressed using a $\mathrm{KBr}$-Press laboratory hydraulic press. Before compression, the surfaces of the die and punch were lubricated with magnesium stearate. All the preparations were stored in airtight containers at room temperature for further study.

Evaluation of tablets: The tablets of each batch were evaluated for thickness, diameter, weight variation, hardness, and friability. Randomly collected 20 tablets of each batch were evaluated for thickness and diameter by digital slide calipers and then they undergo weight variation test. Each tablet was individually weighed. The average weight and standard deviation of 20 tablets was calculated. Then the tablets were tested by Monsanto hardness tester. Another twenty tables were weighed and placed in the Electorlab friabilator and the apparatus was rotated at $25 \mathrm{rpm}$ for 4 minutes. After revolutions the tablets were dedusted and weighed again. The percentage of friability was measured using the formula of Gendle et al., (2010):

$\% \mathrm{~F}=\left\{1-\left(\mathrm{W}_{\mathrm{t}} / \mathrm{W}\right)\right\} \times 100$

Where, $\% \mathrm{~F}=$ friability in percentage

$\mathrm{W}=$ Initial weight of tablets

$\mathrm{W}_{\mathrm{t}}=$ Weight of tablets after revolution

The average of diameter, thickness, hardness of 20 tablets of each formulation along with the weight variation and \% friability of each batch have been provided on Table 2 .

Table 1. Composition of different formulations of Simvastatin floating tablet (in mg)

\begin{tabular}{lcccccc}
\hline \multicolumn{1}{c}{ Ingredients } & F-1 & F-2 & F-3 & F-4 & F-5 & F-6 \\
\hline Simvastatin & 40 & 40 & 40 & 40 & 40 & 40 \\
Methocel K4M & 100 & 120 & 140 & 160 & 140 & 200 \\
Povidone K-30 & 40 & 40 & 40 & 40 & 40 & 40 \\
Ludipress & 210 & 190 & 170 & 150 & 130 & 110 \\
NaHCO $_{3}$ & 60 & 60 & 60 & 60 & 60 & 60 \\
Citric acid & 40 & 40 & 40 & 40 & 40 & 40 \\
Mg. Stearate & 5 & 5 & 5 & 5 & 5 & 5 \\
Talc & 5 & 5 & 5 & 5 & 5 & 5 \\
Total weight & 500 & 500 & 500 & 500 & 500 & 500 \\
\hline
\end{tabular}

Determination of in vitro floating lag time: The in vitro buoyancy was determined by floating lag time and total floating time, as per the method described by Dave et al., (2004) in a $250 \mathrm{~mL}$ beaker containing $0.1 \mathrm{~N} \mathrm{HCl}, \mathrm{pH}$ 1.2, maintained at $37 \pm 0.5^{\circ} \mathrm{C}$ in a water bath. Their physical state was observed for $24 \mathrm{~h}$. The time required for the tablet to rise to the surface and float was determined as floating lag time and total duration of time by which dosage form remain buoyant is called total floating time.

Determination of swelling index: The swelling index of tablets was determined in $0.1 \mathrm{~N} \mathrm{HCl}(\mathrm{pH} 1.2)$ at $37^{\circ} \mathrm{C}$ temperature. The swollen weight of the tablet was determined at predefined time intervals over a period of 5 hr. The swelling index (SI) is expressed as a percentage and was calculated from the following equation (Deshpande et al., 1997). 
Weight of tablet at time ( $\mathrm{t})$ - Initial weight of tablet $\mathrm{SI}=$ Initial weight of tablet

Compatibility assuring test using Fourier Transformer Infrared (FT-IR) spectroscopy: FT-IR spectrophotometer (IR-Prestige 21, Shimadzu, Japan) was used to obtain the spectra of the Simvastatin and the formulation $\mathrm{F}-1$ by $\mathrm{KBr}$ disc method. Polystyrene disc was used as scanning reference before measurement of samples by FT-IR spectrophotometer in the range of wave number 2000-400 $\mathrm{cm}^{-1}$. The samples were prepared in a smooth agate mortar and compressed into a disc of 13-mm diameter using hydraulic press.

In vitro dissolution studies: The release rate of Simvastatin from floating tablet was determined by Dissolution Tester Veego VDA-8DR using paddle apparatus. The dissolution test was performed using 900 $\mathrm{ml} 0.1 \mathrm{~N} \mathrm{HCl}$ with $1 \%$ sodium lauryl sulphate at $37^{\circ} \mathrm{C} \pm$ $0.5^{\circ} \mathrm{C}$ and $75 \mathrm{rpm}$. At each hour interval, aliquots of $10 \mathrm{ml}$ were withdrawn from the dissolution medium and the amount was replaced with fresh medium to maintain the volume constant. The samples were filtered through a Whatmaan filter paper and diluted to a suitable concentration with dissolution media for the study. The absorbance of the solutions was measured at $238 \mathrm{~nm}$ for Simvastatin by using a Shimadzu mini UV-1240 UV/Visible spectrophotometer (Shimadzu, Japan). Cumulative percentage of drug release was calculated using an equation obtained from standard curve.

In vitro drug release kinetic studies: The dissolution profiles of all the batches were fitted to zero-order, (Gibaldi and Feldman, 1967) by plotting cumulative amount of drug release versus time, first-order by plotting log cumulative percentage of drug remaining versus time, Higuchi, (Higuchi, 1963) by plotting cumulative percentage of release versus square root of time, Korsmeyer-Peppas equation models (Korsmeyer et al., 1983; Peppas, 1985) by plotting log cumulative percentage of drug released versus log time and HixonCrowell model (Hixson and Crowell, 1931) by plotting cubic root of percent releases (cubic root of initial drug load minus cubic root of \% drug remaining) against time. To characterize the drug release rate in different experimental conditions, $\mathrm{T}_{25 \%}, \mathrm{~T}_{50 \%}$ and $\mathrm{T}_{80 \%}$ were calculated from dissolution data according to the following equations:

$$
\begin{aligned}
\mathrm{T}_{25 \%} & =(0.25 / \mathrm{k})^{1 / \mathrm{n}} \\
\mathrm{T}_{50 \%} & =(0.5 / \mathrm{k})^{1 / \mathrm{n}} \\
\mathrm{T}_{80 \%} & =(0.8 / \mathrm{k})^{1 / \mathrm{n}}
\end{aligned}
$$

Mean dissolution time (MDT) value was used to characterize the drug release rate from the dosage form and the retarding efficiency of the polymer. A higher value of MDT indicates a higher drug retaining ability of the polymer and vice-versa. Mean Dissolution Time (MDT) was also calculated by the following equation (Mockel and Lippold, 1993).

MDT $=(n / n+1) \cdot K^{-1 / n}$

\section{Results and Discussion}

Floating tablets of Simvastatin were prepared by direct compression method. The tablets were evaluated for weight variation, friability, hardness, diameter and thickness for all the formulations (F-1 to F-6). No significant difference was observed in the weight of individual tablets from the average weight. The hardness of tablets of all formulations was in acceptable limits $\left(11.06 \mathrm{~kg} / \mathrm{cm}^{2}\right.$ to $\left.11.65 \mathrm{~kg} / \mathrm{cm}^{2}\right)$. All the formulation showed \% friability less than $1.0 \%$, which indicates the ability of tablets to withstand shocks. No significant difference was observed in the thickness of individual tablet from the average.

Table 2. Physical characterization of prepared tablets

\begin{tabular}{cccccc}
\hline Formulation & $\begin{array}{c}\text { Diameter } \\
(\mathrm{mm})\end{array}$ & $\begin{array}{c}\text { Thickness } \\
(\mathrm{mm})\end{array}$ & $\begin{array}{c}\text { Hardness } \\
\left(\mathrm{Kg} / \mathrm{cm}^{2}\right)\end{array}$ & $\begin{array}{c}\text { Average } \\
\text { weight }(\mathrm{mg})\end{array}$ & $\begin{array}{c}\text { Friability } \\
(\%)\end{array}$ \\
\hline F-1 & $13.07 \pm 0.0023$ & $2.77 \pm 0.01$ & $11.6 \pm 0.05$ & $500 \pm 1.2$ & 0.198 \\
F-2 & $13.06 \pm 0.0024$ & $2.81 \pm 0.02$ & $11.5 \pm 0.06$ & $500 \pm 0.95$ & 0.130 \\
F-3 & $13.07 \pm 0.0023$ & $2.69 \pm 0.02$ & $11.1 \pm 0.04$ & $500 \pm 0.49$ & 0.039 \\
F-4 & $13.08 \pm 0.0019$ & $2.65 \pm 0.01$ & $11.4 \pm 0.03$ & $500 \pm 0.07$ & 0.031 \\
F-5 & $13.08 \pm 0.0020$ & $2.79 \pm 0.01$ & $11.5 \pm 0.04$ & $500 \pm 0.48$ & 0.194 \\
F-6 & $13.07 \pm 0.0022$ & $2.80 \pm 0.01$ & $11.4 \pm 0.08$ & $500 \pm 0.08$ & 0.069 \\
\hline
\end{tabular}

IR spectra of pure Simvastatin and the formulation F1 (Figure 2) were found to be identical which indicated no interaction between Simvastatin and used excipients at the incorporated ratio. Rather the spectra of formulation F-1 was clearly composed of identical peaks of Simvastatin and its characteristic peaks were not affected by the presence of excipients used in the formulation. In addition, no degradation during manufacturing process was found from IR spectra.

Buoyancy lag time and total floating time were determined and the results are shown in Table 3. Upon observation for more than 24 hours, it can be concluded that all formulations had floating lag times below 3 minutes and constantly floated on acidic medium for more than 5 hours. F-5 and F-6 showed good buoyancy lag time is $46 \mathrm{sec}$ and $27 \mathrm{sec}$ respectively and total floating time was more than $22 \mathrm{hr}$. 


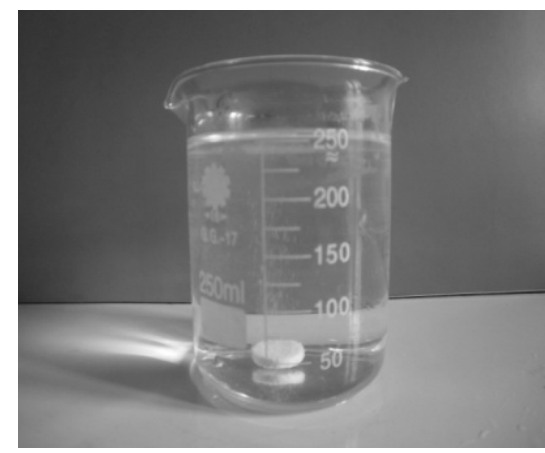

At zero time

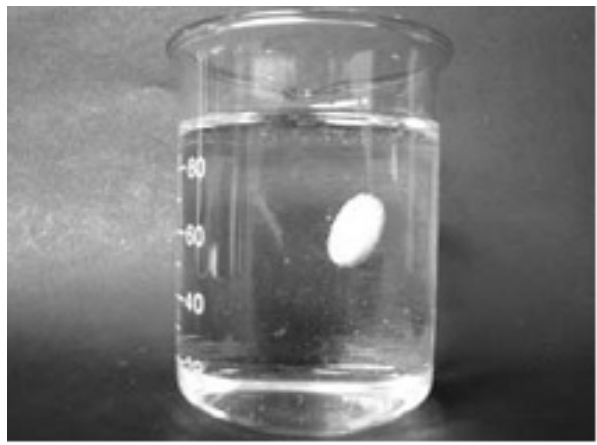

After 25 seconds

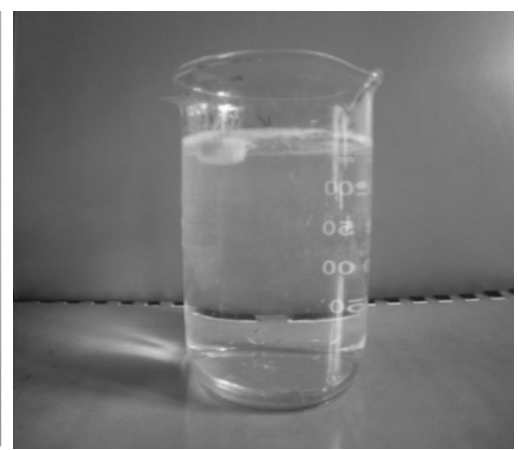

After 27 seconds

Figure 1. Pictorial presentation of in vitro floating behavior of a representative tablet based on Methocel K4M

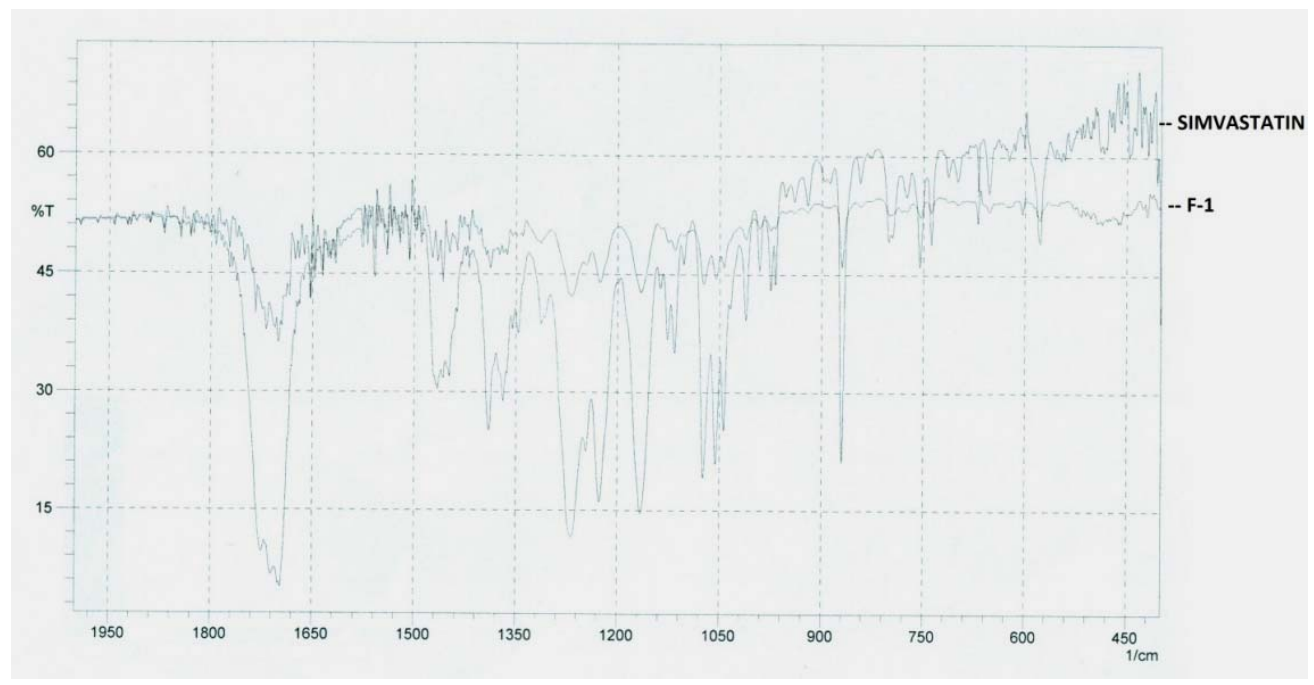

Figure 2. FT-IR spectra of Simvastatin (large peaks) and formulation F-1

Table 3. In vitro Buoyancy studies

\begin{tabular}{ccc}
\hline Formulation & $\begin{array}{c}\text { Buoyancy Lag } \\
\text { Time (Sec) }\end{array}$ & $\begin{array}{c}\text { Total Floating } \\
\text { Time (Hour) }\end{array}$ \\
\hline F-1 & 165 & $>5 \mathrm{hrs}$ \\
F-2 & 150 & $>5 \mathrm{hrs}$ \\
F-3 & 115 & $>10 \mathrm{hrs}$ \\
F-4 & 76 & $>19 \mathrm{hrs}$ \\
F-5 & 46 & $>22 \mathrm{hrs}$ \\
F-6 & 27 & $>24 \mathrm{hrs}$ \\
\hline
\end{tabular}

The swelling indexes for all formulation F-1 to F-6 are shown in Table 4. Swelling indexes have a direct relationship on tablet floating. Initially the index had found to rise quickly due to the rapid water intake of the water soluble matrix. This water intake made the matrix swell and thus reduced the bulk density that is responsible for buoyancy. The total floating time hence, depends on the decrease of bulk density. An increasing order of swelling index was found for formulations (F-4, F-5 and F-6) having higher total floating time. On the other hand, the formulations F-1, F-2 and F-3 were found to have decreasing order of swelling index as the matrix was eroding and getting dissolved in the medium with time progression. As a result these formulations had higher floating lag time, lower total floating time and burst release within hours in the in vitro dissolution study. Similar findings were reported by Sharmin et al., (2011) who formulate floating tablet of Atorvastatin using Methocel K4M and Methocel K 15MCR. 
Table 4. Swelling index studies

\begin{tabular}{cccccc}
\hline Formulation & \multicolumn{5}{c}{ Swelling Index (\%) } \\
& $1 \mathrm{hr}$ & $2 \mathrm{hr}$ & $3 \mathrm{hr}$ & $4 \mathrm{hr}$ & $5 \mathrm{hr}$ \\
\hline F-1 & 41.08 & 29.75 & 14.00 & 5.84 & -2.47 \\
F-2 & 79.40 & 46.17 & 32.01 & 17.70 & 8.59 \\
F-3 & 95.71 & 92.24 & 84.14 & 69.34 & 42.63 \\
F-4 & 110.47 & 128.74 & 162.41 & 177.54 & 180.49 \\
F-5 & 120.44 & 136.08 & 157.14 & 180.70 & 184.22 \\
F-6 & 126.24 & 142.70 & 161.42 & 179.64 & 185.25 \\
\hline
\end{tabular}

The cumulative percent drug release data obtained for formulations F-1 to F-6 is plotted in Figure 3. Formulations F-1 to F-6 containing different concentrations of Methocel K4M, were found to retard the drug release as a function of polymer loading. F-1, F-2 and F-3 were found to release $92.90 \%, 75.16 \%$ and $51.70 \%$ of incorporated drug, respectively after 8 hours of dissolution in acidic medium. These formulations were designed using the polymer at 20, 24 and 28\% w/w concentration. Later on, addition of more polymer in the formulations rendered the matrix more capable of retarding the release of Simvastatin. Having 32, 36 and $40 \% \mathrm{w} / \mathrm{w}$ concentration of polymer in the matrix showed $40.90 \%, 37.28 \%$ and $32.59 \%$ drug release after 8 hours from formulation F-4, F-5 and F-6 respectively. Similar rate retarding ability of Methocel K4M was reported by Mishra et al., (2006) who claimed that higher viscosity, high molecular weight, slower rate of erosion and higher swelling ability render the polymer the ability to retard the rate of drug release.

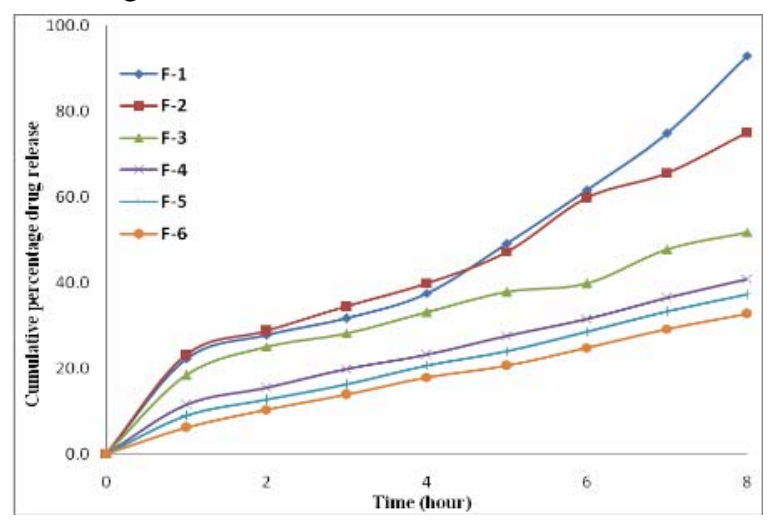

Figure 3. Zero order plot of Simvastatin from floating tablets

Methocel K4M has been well known to retard the drug release by swelling in aqueous media. A polymer's ability to retard the drug release rate is related to its viscosity. Processing factors including particle size, hardness, porosity and compressibility index, etc. can also affect the release rate of drug from tablets (Ebube et al., 1997). The hydration rate of HPMC depends on the nature of the substituents like hydroxypropyl group content. Hence, Methocel K4M was used because it forms a viscous gel in contact with aqueous media, which may be useful in controlled delivery of drug (Gao et al., 1996). Thus from the cumulative data, it can be concluded that rate controlling ability of Methocel K4M depends on its capacity of forming viscous gel and hence, rate retardation is achieved by using the polymer at the higher quantity in the matrix.

The in vitro drug release data obtained were fitted to kinetic models viz., Zero order (Figure 3), First order (Figure 4), Higuchi (Figure 5), Hixson-Crowell equations (Figure 6) and Korsmeyer-Peppas (Figure 7) to know the pattern of drug release and mechanism of drug release from the matrix tablets. All six formulations (F-1 to F-6) showed fairly good linearity with zero order plot having $\mathrm{R}^{2}$ value ranging from 0.930 to 0994 (Table 5). Formulation F-1 and F-2 containing lower quantity of rate retarding polymer in the matrix; had been found best fit to zero order that indicates that the rate of drug release behavior is independent of its initial loading.

Good correlation coefficients (except formulation F1) observed for the kinetic parameters based on the first order model equation were mainly due to the drug release mechanism (Figure 4). First order plot for formulation F-2 to F-6 showed good linearity. Particularly formulation F-4 and F-5 showed best fitting to first order plot. This indicates that the amount of drug released is dependent on the matrix drug loading.

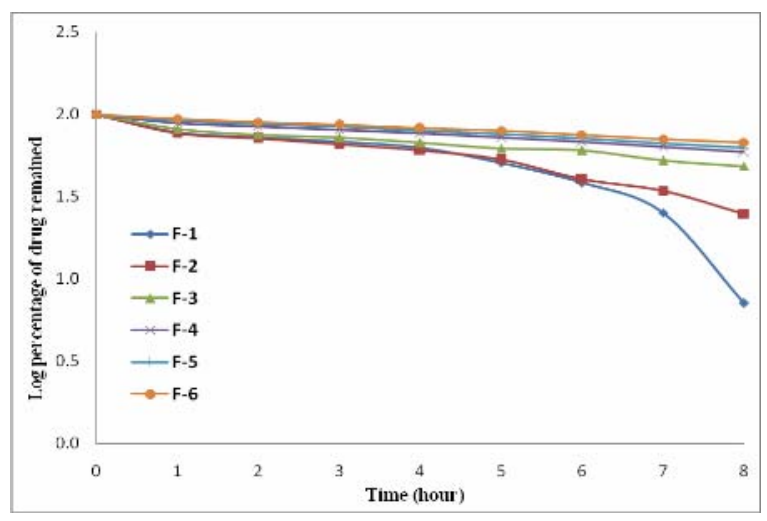

Figure 4. First order plot of Simvastatin release from floating matrix tablets 
In vitro drug dissolution data of all formulations except F-1, showed good linearity to Higuchi model $\mathrm{R}^{2}$ values ranging from 0.876 to 0.986 (Figure 5). Incorporation of more the polymer in the matrix rendered the drug release profile linear to Higuchi plot. Formulation F-3 had been found to best fit with Higuchi model having $\mathrm{R}^{2}$ value 0.986 that confirmed that the drug were released by mainly diffusion. The release profile of Simvastatin from all these formulations displayed not a good fitting to Hixson-Crowell cube root model of drug release conferred that dissolution was not the predominant mechanism for drug release (Figure 6). The regression coefficient values for Hixson-Crowell cube root model were found only from 0.595 to 0.737 .

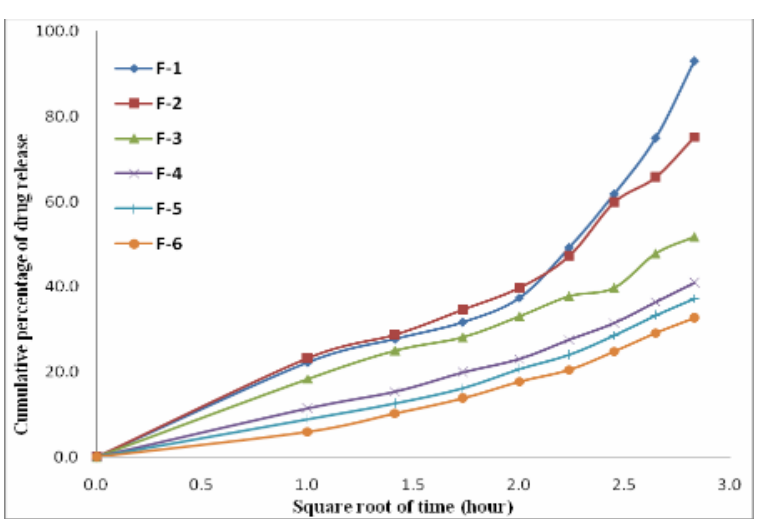

Figure 5. Higuchi's plot of Simvastatin release from floating matrix tablets

To confirm the release mechanism of Simvastatin from floating matrix, the data were fitted to KorsmeyerPeppas equation. All the formulations showed good linearity (0.891 to 0.997) with slope $n$ values ranging from 0.484 to 0.804 indicating that the release mechanism was non-Fickian or anomalous release $(0.45<n<0.89)$. It can be postulated that the release from the hydrophilic matrix was dependent on both drug diffusion and polymer relaxation, which appears to indicate a coupling of diffusion and erosion mechanisms so called anomalous diffusion (Sato et al., 1997). Formulation F-6 was found best fit with the model having the $\mathrm{R}^{2}$ value 0.997 .

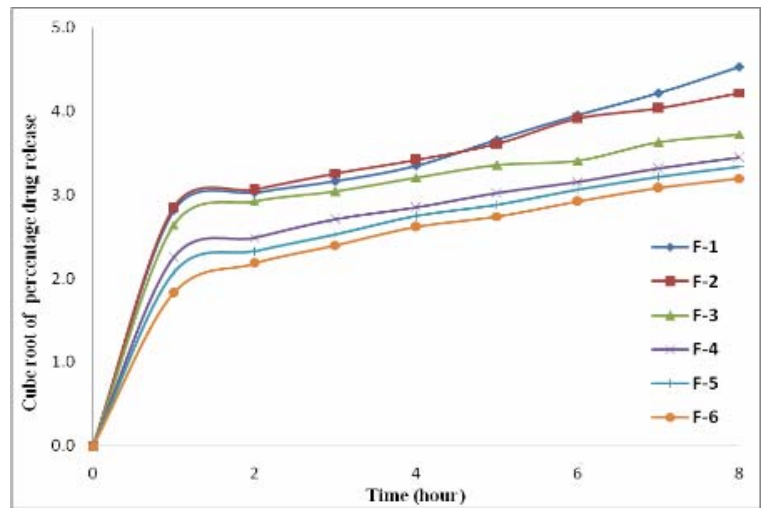

Figure 6. Hixson Crowell plot of Simvastatin release from floating matrix tablets

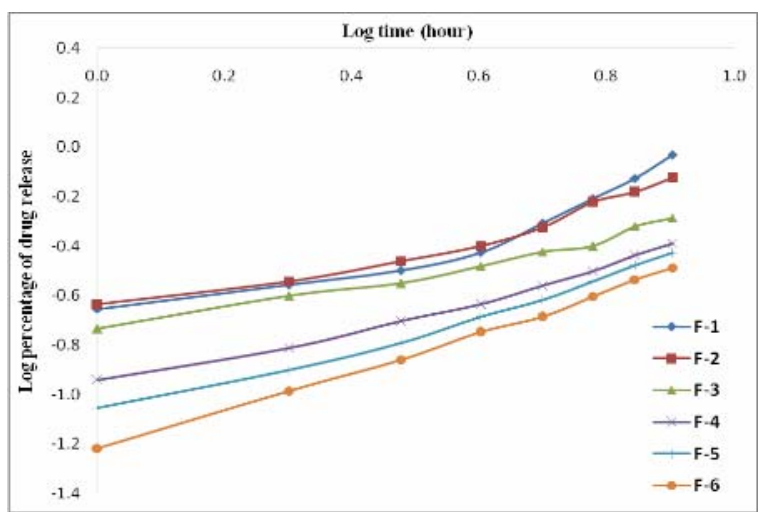

Figure 7. Korsmeyer's plot of Simvastatin release from floating matrix tablet

Table 5. In vitro release kinetic data for the prepared formulations of Simvastatin.

\begin{tabular}{cccccccccccc}
\hline $\begin{array}{c}\text { Formu- } \\
\text { lation }\end{array}$ & \multicolumn{2}{c}{ Zero Order } & \multicolumn{2}{c}{ First Order } & \multicolumn{2}{c}{ Highuchi } & \multicolumn{2}{c}{ Korsmeyer } & \multicolumn{2}{c}{ Hixon-Crowell } \\
& $\mathrm{K}_{\mathrm{o}}$ & $\mathrm{R}^{2}$ & $\mathrm{~K}_{1}$ & $\mathrm{R}^{2}$ & $\mathrm{~K}_{\mathrm{h}}$ & $\mathrm{R}^{2}$ & $\mathrm{n}$ & $\mathrm{R}^{2}$ & $\mathrm{~K}_{\mathrm{hc}}$ & $\mathrm{R}^{2}$ \\
\hline F-1 & 10.240 & 0.961 & -0.112 & 0.768 & 29.810 & 0.876 & 0.677 & 0.891 & 0.411 & 0.724 \\
F-2 & 8.377 & 0.967 & -0.067 & 0.954 & 25.360 & 0.954 & 0.572 & 0.941 & 0.374 & 0.656 \\
F-3 & 5.566 & 0.930 & -0.035 & 0.966 & 17.470 & 0.986 & 0.484 & 0.976 & 0.319 & 0.595 \\
F-4 & 4.643 & 0.976 & -0.026 & 0.987 & 14.110 & 0.970 & 0.616 & 0.982 & 0.310 & 0.666 \\
F-5 & 4.369 & 0.989 & -0.023 & 0.993 & 13.080 & 0.954 & 0.701 & 0.985 & 0.310 & 0.705 \\
F-6 & 3.924 & 0.994 & -0.020 & 0.996 & 11.650 & 0.944 & 0.804 & 0.997 & 0.306 & 0.737 \\
\hline
\end{tabular}


MDT value represents the retarding efficacy of polymer incorporated in the matrix system. $\mathrm{T}_{25 \%}, \mathrm{~T}_{50 \%}$, $\mathrm{T}_{80 \%}$ and MDT values are depicted in the Figure 8 . The values showed a gradual increase in the retaining ability on increasing the polymer loading. Formulation F-1 and F2 were found to have a very lower level of MDT value of $5.02 \mathrm{hr}$ and $5.87 \mathrm{hr}$ respectively that indicated little sustainability of the polymer in the formulations. But on addition of more polymer, the matrix was found to gain the capacity to retain the incorporated drug as the MDT value of formulation F-3 and F-6 were $11.78 \mathrm{hr}$ and 15.01 hr respectively. Though after using the polymer at $32 \%$ of the matrix and more (F-4 to F-6), the $\mathrm{T}_{25 \%}, \mathrm{~T}_{50 \%}, \mathrm{~T}_{80 \%}$ and MDT values increased a little.

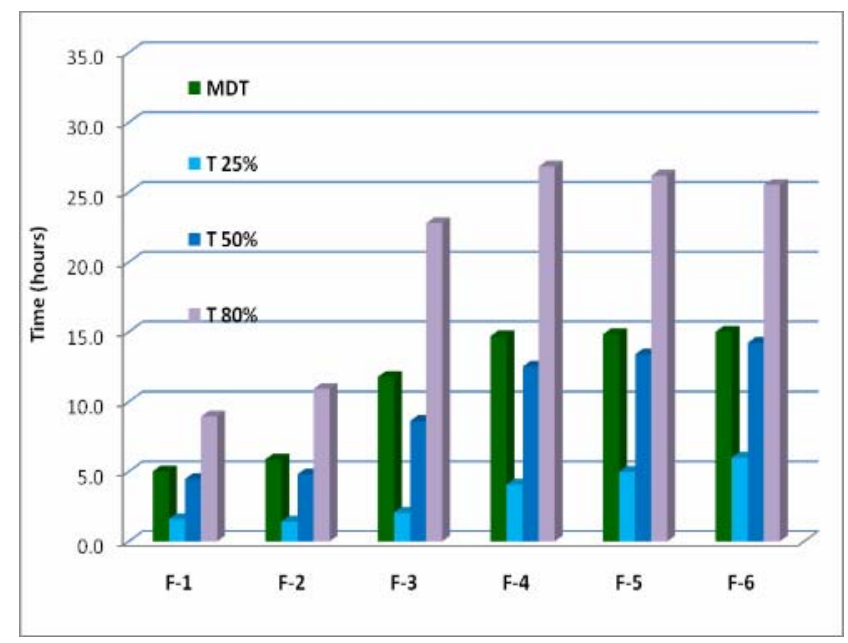

Figure 8. Comparison of MDT, $\mathrm{T}_{25 \%}, \mathrm{~T}_{50 \%}$ and $\mathrm{T}_{80 \%}$ values of different formulation (in hour)

\section{Conclusion}

Gastro retentive floating tablets of Simvastatin were prepared using hydrophilic cellulose derivative Methocel K4M. All the formulations were able to float instantaneously and kept floating for more than 5 hours to more than 24 hours with controlling the release rate throughout the time. Methocel K4M was found successful in controlling the drug release rate as a function of incorporated polymer in the matrix. The drug release mechanism was found non fickian; dependent on both drug diffusion and polymer relaxation. An effective floating tablet of Simvastatin can be prepared by using the polymer at different ratio to modify the release kinetics and floating behavior in order to design a more appropriate drug delivery system.

\section{References}

Asif M., Yasir M., Bhattacharya A. and Bajpai M. 2010. Formulation and evaluation of gastroretentive dosage form for Fluvastatin Sodium. Pharm. Glob. Int. J. Comp. Pharm. 01, 1-4.

Choi B.Y., Park H.J., Hwang S.J. and Park J.B. 2002 Preparation of alginate beads for floating drug delivery system: effects of CO2 gas-forming agent. Int. J. Pharm. 239, 81-91.

Dave B.S., Amin A.F. and Patel M.M. 2004. Gastroretentive drug delivery system of ranitidine hydrochloride: formulation and in vitro evaluation. AAPS Pharm. Sci. Tech. 5, 30-34.

Deshpande A.A., Shah N.H. and Rhodes C.T. 1997. Development of a novel controlled-release system for gastric retention. Pharm. Res. 14, 815-819.

Ebube N.K., Hikal A., Wyandt C.M., Beer D.C., Miller L.G. and Jones A.B. 1997. Sustained release of acetaminophen from heterogeneous matrix tablets: influence of polymer ratio, polymer loading, and co-active on drug release. Pharm. Dev. Technol. 2, 161-170.

Ganesh S.K., Nass C.M. and Blumenthal R.S. 2003. Antiatherosclerotic effects of statins: lessons from prevention trials. J. Cardiovasc. Risk. 10,155-159.

Gao P., Skoug J.W., Nixon P.R., Ju T.R., Stemm N.L. and Sung K.C. 1996. Swelling of hydroxypropyl methylcellulose matrix tablets. 2. Mechanistic study of the influence of formulation variables on matrix performance and drug release. J. Pharm. Sci. 85, 732-740.

Gendle R., Kaushik B., Verma S., Patel R., Singh S.K. and Namdeo K.P. 2010. Formulation and evaluation of sustained release matrix tablet of Tramadol Hcl. Int. J. Chem. Tech. Res. 2, 4-10.

Gibaldi M. and Feldman S. 1967. Establishment of sink conditions in dissolution rate determinations- theoretical considerations and application to non-disintegrating dosage forms. J. Pharm. Sci. 56, 1238- 1242.

Higuchi T. 1963. Mechanism of sustained release medication: theoretical analysis of rate of release of solid drugs dispersed in solid matrices. J. Pharm. Sci. 52, 1145-1149.

Hixson A.W. and Crowell J.H. 1931. Dependence of reaction velocity upon surface and agitation: I-theoretical consideration. Int. Eng. Chem. 23, 923-931.

Korsmeyer R.W., Gumy R., Doelker E., Buri P. and Peppas N.A 1983. Mechanism of solute release from porous hydrophilic polymer. Int. J. Pharm. 15, 25-35. 
Kulkarni A. and Bhatia M. 2009. Development and evaluation of regioselective bilayer floating tablets of Atenolol and Lovastatin for biphasic release profile. Iranian J. Pharm. Res. 8, 15-25

LaRosa J.C. 2000. Statins and risk of coronary heart disease. $J$. American Med. Assoc. 283, 2935-2936.

Mishra B., Bharti V.B., Singh P.N., Kumar P. 2006. Development and In-vitro evaluation of oral sustained release formulation of Tramadol Hydrochloride. Acta Phar. Sci. 48, 153-166.

Mockel J.E. and Lippold B.C. 1993. Zero order release from hydrocolloid matrices. Pharm. Res. 10, 1066-1070.

Mundy G., Garrett R., Harris S., Chan J., Chen D., Rossini G., Boyce B., Zhao M. and Gutierrez G. 1999. Stimulation of bone formation in vitro and in rodents by statins. Science. 286, 1946-1949.
Peppas N.A. 1985. Analysis of Fickian and non-Fickian drug release from polymers. Pharm. Acta. Helv. 60, 110-111.

Rouge N., Buri P. and Doelker E. 1996. Drug absorption sites in the gastrointestinal tract and dosage forms for site-specific delivery, Int. J. Pharm. 136, 117- 139.

Sharmin F., Masum M.A.A., Islam S.M.A. and Reza M.S. 2011. Preparation and evaluation of gastro retentive floating tablets of Atorvastatin Calcium. Dhaka Univ. J. Pharm. Sci. 10, 79-85.

Sato H., Miyagawa Y., Okabe T., Miyajima M. and Sunada H. 1997. Dissolution mechanism of diclofenac sodium from wax matrix granules. J. Pharm. Sci. 86, 929-934.

Singh B.M. and Kim K.H. 2000. Floating drug delivery systems: an approach to oral controlled drug delivery via gastric retention. J. Control. Rel. 63, 235-259. 